\title{
Ein Schminkgefäß und ein General
}

DOI 10.1515/zaes-2016-0013

Summary: The cosmetic vase Durham EG3995, probably from the Early Dynastic period, was reused by a high military commander as a votive in the time of Apries. In its inscription, an anonymous person asks Sakhmet/Hathor, possibly during a ceremony by which the vessel was consecrated for use in the temple, to reward the General for his donation with royal favour and a good reputation.

The military title $m r$ mnfyt of the donor and the meaning of $m n f 3 t / m n f y t$ is discussed: the mnfyt were probably troops transported by ship.

Keywords: consecration - military title - reuse - stone vessel

\section{Das Schminkgefäß Durham EG3995}

Das Steingefäß im Oriental Museum in Durham stammt aus der ehemaligen Sammlung Alnwick Castle (frühere Nummer N1378) $)^{1}$ des $4^{\text {th }}$ Duke of Northumberland und muss vor 1865 erworben worden sein. Weiter ist über seine Herkunft nichts bekannt.

Es ist ein bauchiges Gefäß aus schwarz-weißem Serpentin mit flachem Boden und einer breiten, deutlich abgesetzten Lippe. Die beiden gleichfalls recht breiten Schnurösen sind durchbohrt. Das Gefäß ist $11,0 \mathrm{~cm}$ hoch und an den Henkeln 17,5 cm breit (ohne Henkel 15,5 cm). Das Gefäß ist gut erhalten; kleinere Beschädigungen werden vom Museum folgendermaßen beschrieben: \footnotetext{
Alnwick Castle, London 1880, 174-5.

Prof. Dr. Karl Jansen-Winkeln: Freie Universität Berlin Ägyptologisches Seminar Fabeckstr. 23-25, 14195 Berlin, Germany

E-Mail: jawinkel@zedat.fu-berlin.de
}

„Large chip in one side from the foot to almost half way to the handle. There are two smaller chips in the same side of the foot. One handle has a large cut mark along the underside and an abrasion in the centre, the other is chipped at one end and has

11378 ist die Nummer, unter der das Stück zuerst publiziert worden ist, s. S. Birch, Catalogue of the Collection of Egyptian Antiquities at some brown surface deposits. The rim is chipped in 5 places along the outside edge and 3 on the inside edge. There are some surface abrasions overall, especially over the inscription.“

Ähnlich geformte Steingefäße sind nicht selten und kommen vor allem in frühgeschichtlicher Zeit vor, allerdings meist deutlich kleiner² ${ }^{2}$ es gibt aber auch durchaus größere Exemplare ${ }^{3}$.

Wenn das Gefäß aus Memphis stammen sollte, wie es die Weihung durch Sachmet (s.u.) zumindest nahelegt, wäre es leicht möglich, dass es ein wiederbenutztes altes Objekt war. Wie allein der Fund in der Stufenpyramide ${ }^{4}$ bewiesen hat, waren derartige Gefäße aus ältester Zeit in sehr großen Mengen vorhanden, und es gab sicher auch während der 26. Dynastie Leute, die wußten, wo so etwas zu finden war ${ }^{5}$. Gegen Ende der 22. Dynastie ist ein recht ähnliches Gefäß für den König Nimlot (D) beschriftet worden, dass angeblich aus der Frühzeit oder der 3. Dynastie stammt ${ }^{6}$.

Dennoch wird man kaum grundsätzlich davon ausgehen können, dass Gefäße von archaischem Aussehen, die in deutlich späteren Kontexten vorkommen, ausnahmslos alt sind und wiederverwendet wurden ${ }^{7}$. Auch im Fall des Gefäßes in Durham kann man nicht ausschließen, dass es

2 Vgl. etwa B. Aston, Stone Vessels, SAGA 5, Heidelberg 1994, 79; 91 (Nr. 3); J. Vandier d’Abbadie, Catalogue des objets de toilette égyptiens, Paris 1972, 116-117, Nr. 472; 474-476; 484; A. el-Khouli, Egyptian Stone Vessels, predynastic period to dynasty III, Mainz 1978, I, 327331; pl.84; S. Hodjash, Ancient Egyptian Vessels in the State Pushkin Museum of Fine Art, Moscow, Baltimore 2005, 21; pl.4 (Nr. 32); R. und D. Klemm, Die Steine der Pharaonen, München 1981, 9; Abb. 4 (München ÄS 1596). Zur bisherigen Literatur zu Steingefäßen s. den Überblick von P. Vlčková, Stone Vessels from the Mortuary Complex of Raneferef at Abusir (Abusir XV), Prag 2006, 9-15.

3 Z. B. P. Günther; Wellauer, R., Ägyptische Steingefäße der Sammlung Rudolph Schmidt Solothurn, Zürich 1988, 29-32; Taf.31-32 (Nr.54-56; 61-62); F.W. von Bissing, Steingefäße, CG, Leipzig 1907, 23-24; Taf.IV (Kairo CG 18169); Ch. Lilyquist, Egyptian Stone Vessels, New York 1995, 43; 109 (104: Kairo SR 3481).

$4 \mathrm{PM} \mathrm{III}^{2}$, 402-5; J.-Ph. Lauer, La pyramide à degrés, III, Kairo 1939; IV, 1959; V, 1965; id., ASAE 34, 1934, 54-62; id., Les pyramides à degrés, BdE 39, Kairo 1962, 91-98.

5 Vgl. auch P. Lacovara, AJA 101, 1997, 781.

6 L. Sist, Museo Barracco. Arte egizia, Rom 1996, 61-2 (3. Dynastie); G. Careddu, Museo Barracco di scultura antica. La collezione egizia, Rom 1985, 43-44; 59; Taf. (Nr. 51); L. Bongrani Fanfoni, OrAnt 26, 1987, 65-71; Tav. 2-3; M. Becker, Identität und Krise, BSAK 13, Hamburg 2012, 143-4 (frühdynastisch oder späte Kopie); vgl. auch R. Meffre, D’Hérakléopolis à Hermopolis, Paris 2015, 137-8, n.279.

7 Vgl. Lilyquist, Egyptian Stone Vessels, 10-12. 
sich um ein Gefäß der 26. Dynastie handelt, einer Zeit, die sich ja gern an alten Vorbildern orientiert hat. Handwerker mit der Fähigkeit, Steingefäße in vollendeter Form herzustellen, hat es jedenfalls auch im 1. Jahrtausend in Ägypten genügend gegeben, wie sich nicht zuletzt in der 27. Dynastie zeigt, wo ägyptische Steingefäße in großer Zahl ins persische Reich geliefert wurden ${ }^{8}$. Da aber die „surface abrasions" vorwiegend an der Fläche der Inschrift feststellbar sind (s. o.), spricht das wohl eher dafür, dass der Text nachträglich eingeschnitten worden ist.

Steingefäße dieser Art dienten hauptsächlich als „Salbgefäße und Behälter für Schminke und Parfüm“9. Die scheibenartig abgesetzte Lippe machte es möglich, ein solches Gefäß mit einem Lappen (oder einem Goldblech) abzudecken und diesen Verschluß mit einer Schnur (oder einem Golddraht) $\mathrm{zu}$ befestigen ${ }^{10}$. Ein (ziemlich großes) Gefäß ganz ähnlicher Form wird in einer Szene aus einem Grab des Alten Reiches beim „Abmessen von Salbe mit dem Ölgefäß“ dargestellt (h3t mrht $m$ b $3 q t)^{\mathbf{1 1}}$.

Steingefäße mit längeren Inschriften sind selten ${ }^{12}$, erst recht in der Spätzeit. Auf dem hier behandelten Gefäß Durham EG3995 ist aber eine Seite, der größere Teil des Feldes zwischen den Henkeln, vom oberen Rand bis fast zur Standfläche von rechts nach links mit sechs Kolumnen beschrieben; die Hieroglyphen blicken nach rechts.

Diese Inschrift ist 1880 von S. Birch publiziert und übersetzt worden ${ }^{13}$, aber so fehlerhaft, das ein Verständnis danach nicht möglich war. Immerhin hatte er Kol.1 mit Titeln und Namen richtig wiedergegeben, die danach auch von Chevereau ${ }^{14}$ und Press ${ }^{15}$ zitiert worden sind. In

8 G. Posener, La première domination perse en Egypte, BdE 11, Kairo 1936, 137-151; I. Shaw, „Inscribed Stone Vessels as Symbols of the Egypto-Achaemenid Economic Encounter", in: A. Hudecz, M. Petrik (edd.), Commerce and Economy in Ancient Egypt, Oxford 2010, 113-117.

9 H. Kayser, Ägyptisches Kunsthandwerk, Braunschweig 1969, 18.

10 Loc. cit.

11 T. Pommerening, Die altägyptischen Hohlmaße, Beihefte SAK 10, Hamburg 2005, 314-5.

12 Ein recht ähnlich aussehendes Salbgefäß mit Inschrift ist als Beigabe im Königsgrab von Qatna (Syrien) gefunden worden. Es war ursprünglich „gegeben durch die Gunst“ (djj $m$ hzwt) der AhmesNefertari an einen Schatzmeister namens Nfr-prt, s. A. Ahrens, „A Journey's End: Two Egyptian Stone Vessels with Hieroglyphic Inscriptions from the Royal Tomb of Tell Mišrife/Qaţna“, Ä\&L 16, 2006, 15-36.

13 Birch, Catalogue of the Collection of Egyptian Antiquities at Alnwick Castle, 174-5.

14 P.-M. Chevereau, Prosopographie des cadres militaires égyptiens de la Basse Époque, Antony 1985, 126 (180).

15 D. Pressl, Beamte und Soldaten. Die Verwaltung in der 26. Dynastie in Ägypten (664-525 v. Chr.), Frankfurt a. M. 1998, 319 (S 94). meinen Inschriften der Spätzeit habe ich nach Fotos des Museums schon einen verbesserten Text wiedergegeben ${ }^{16}$.

Die Inschrift ist aufgrund des Materials recht schwierig zu lesen und wegen der Rundungen auch nicht einfach zu fotografieren (Abb. 2-3). Außerdem sind zwei Stellen in Kol. 2 und 4 leicht beschädigt. Ich hoffe aber, anhand der Fotos, die ich vom Museum bekommen habe, nun eine korrekte Abschrift geben zu können (Abb.1).

Der Besitzer bzw. Stifter des Gefäßes, ein General ( $m r$ $m n f y t$, s. u.) namens $\underline{D} h w t j-m$ - $3 h t$, der auch den Rang(titel) einer „Exzellenz“ ( $r p^{c} t h h^{3} t j-$ - $)$ führt, scheint aus anderen Quellen nicht bekannt zu sein.

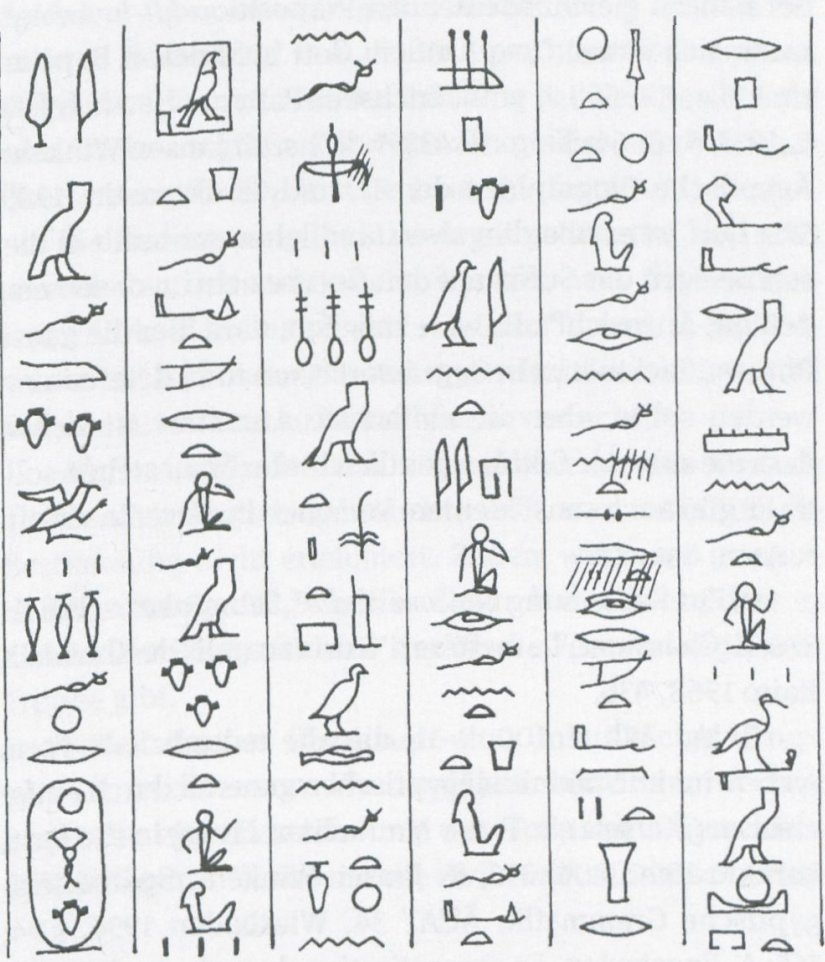

Abb. 1: Abschrift der Inschrift auf dem Schminkgefäß Durham EG3995.

\section{Übersetzung:}

1 Der Erbfürst und Graf, der General ( $m r$ mnfyt) $D h w t j-m$ $3 h t, 2$ indem Sachmet vor ihm befindlich (1) ist:

„Er hat dir Schminke (2) bereitet, so viel du willst (3), und Myrrhen nach 3 Herzenslust (4).

Dein ist der Jubel, den er für dich, seine Herrin, anstimmt.

Mögest du 4 ihm Silber und Schätze geben am Platz des Königs!

16 K. Jansen-Winkeln, Inschriften der Spätzeit, IV: Die 26. Dynastie, Wiesbaden 2014, 1143-4 (60.715). 
Er hat den Rauschtrank dargebracht (5) für 5 Hathor, seine Herrin.

Mögest du die Liebe zu ihm (6) in die Herzen der Menschen geben, 6 seine Beliebtheit in die Herzen (7) des Volkes $(r h y t)$ und seine Gunst zum (König) $W 3 h$-j $b-R^{c}$.“

\section{Anmerkungen:}

1) Die Präposition $h f t$ wird gern (wenn auch nicht ausschließlich) in „feierlichen“ Kontexten verwendet, z. B. wenn man „,or / in Gegenwart / angesichts“ einer Gottheit ist. Ungewöhnlich ist hier aber die sitzende Göttin nach $h f t$. Ein Götterdeterminativ ist sonst einigemale bei der nahezu gleichbedeutenden Präposition $h f t-h r$ belegt, wenn sich jemand „vor“ einem Gott befindet, z. B. pHarris I, 5,4; 7,3; 56b,2, s. W. Erichsen, Papyrus Harris I, BAe 5, 1933, 5; 8; 64; Kairo JE 43359, B,7, s. K. Jansen-Winkeln, Ägyptische Biographien der 22. und 23. Dynastie, 1985, 572. Dort ist es allerdings verständlicher, weil sich in diesen Belegen das Suffix auf den Gott bezieht: „vor seinem/ deinem Angesicht“. Es wäre möglich, dass hier die ganze Phrase „Sachmet ist in Gegenwart“ (von ihm) determiniert werden sollte, aber die einfachste Annahme ist sicher, dass die sitzende Göttin eigentlich hinter Shmt stehen sollte. Es gibt auch sonst kleinere Versehen in dieser Inschrift, s. Anm. 6 und 7.

2) Zur Schreibung von $s m t<s d m$ „Schminke“ s. Wb IV, 370; E. Chassinat, Le mystère d'Osiris au mois de Khoiak II, Kairo 1968, 436.

3) Vgl. Wb II, 100, 9-11; die alte reduplizierte Form $m r r=$ wird im Spätmittelägyptischen generell durch einfaches $m r(j)$ ersetzt, s. P. der Manuelian, Living in the Past, London 1994, 206; 216; K. Jansen-Winkeln, Spätmittelägyptische Grammatik, ÄUAT 34, Wiesbaden 1996, §94; 765; A. Engsheden, La reconstitution du verbe en égyptien de tradition, 400-30 avant J.-C., Uppsala 2003, 42-44; D. Kurth, Einführung ins Ptolemäische, Bd. 2, Hützel 2008, §136-137; J. Lustman, Etude grammaticale du Papyrus Bremner-Rhind, Paris 1999, 70.

4) Wb IV, 533,3 kennt nur $r \check{s} z p j b=$ (mit Suffix). Vielleicht ist auch hier tatsächlich $j b(. t)$ zu lesen und das Possessivsuffix wegen lautlicher Reduktion nicht geschrieben worden. Nach der Präposition $n$ (Kol.2 und 3) und als Subjekt in dj.t "mögest du geben" (Kol. 3 und 5) wird das Suffix . $t$ in dieser Inschrift allerdings geschrieben. Die Phrase würde aber zweifellos auch in der Form ,entsprechend dem, was das Herz annimmt“ (statt „was dein Herz annimmt“) einen akzeptablen Sinn ergeben.

5) Präteritales $w d n . f$ wäre zwar duchaus möglich, denn in der Spätzeit werden $s \underline{d m} . f$ und $s \underline{d m} . n . f$ als präteritale Hauptsatzformen nicht selten unterschiedslos gebraucht, vgl. Engsheden, La reconstitution du verbe, 158 ff. Parallel zu jrj.n.f in Kol.2 ist die Verbform aber vermutlich eher als $w d n<. n>f$ zu verstehen, bei Verben auf $n$ ist die Möglichkeit einer Verwechslung besonders naheliegend, s. Jansen-Winkeln, Spätmittelägyptische Grammatik, $§ 92 ; 102$.

6) Das .f von mrwt.f in stark verkleinerter Form über dem Rücken des $m$ eingefügt, vermutlich nachträglich.

7) Die Schreibung von $j b w$ mit nur zwei Herzen kann eigentlich nur ein Fehler sein; hier wird man keinen Hintersinn vermuten dürfen.

Die kleine Inschrift auf diesem Gefäß hat eine einfache Struktur: Zunächst wird der Besitzer bzw. Stifter genannt und die Situation, für die die folgende „Ansprache“ an die Göttin bestimmt ist. Dann folgen je zweimal das, was er für die Göttin getan hat und die dafür erwünschte Belohnung: Gabe und (erbetene) Gegengabe.

Da ein Steingefäß dieser Form und Größe in der Regel für Salbe oder Schminke bestimmt war und in der Inschrift Augenschminke $(s d m)$ genannt wird, außerdem von Jubel und Rauschtrank für die Göttin die Rede ist, sollte das Gefäß etwas mit Götterfesten der Sachmet/Hathor zu tun haben. Es wurde sicher nicht leer, sondern mit Inhalt gestiftet; allerdings ist in der Inschrift von Schminke und Myrrhen die Rede, und beides kann ja nicht in dem einen Gefäß gewesen sein. Zudem will der Stifter auch Rauschtrank dargebracht haben, was ein weiteres (und ganz anderes) Gefäß erfordern würde. Vermutlich hatte der General mehrere Dinge für ein bestimmtes Fest oder für die Tempelausstattung allgemein gestiftet.

Die Frage ist daher, ob dieses Gefäß (mit seinem Inhalt) Teil dieser Stiftung war und selbst (neben anderen) im Kult verwendet wurde, oder ob es ein reines Votivgefäß war, um für diese Gaben (Schminke, Myrrhen und Wein) Belohnung einzufordern. Die Inschrift darauf spricht wohl eher für eine Votivgabe und gegen eine tatsächliche Verwendung im Kult.

Wenn sich der Stifter des Gefäßes, der General $\underline{D} h w t j-$ $m$-3ht, zu Beginn der Inschrift „vor" Sachmet befindet, wird sich das wohl auf die Situation der Übergabe oder Präsentation des Gefäßes beziehen, als es in Gegenwart der Gottheit „geweiht“ wurde. Man wird a priori davon ausgehen dürfen, dass Gegenstände, die im Götterkult verwendet oder (z. B. als Votivgabe) im Tempel aufgestellt waren, nicht ohne alle Umstände von der Werkstatt in den Tempel verfrachtet wurden, sondern in irgendeiner Form sakralisiert oder „geweiht“ werden mußten. Leider ist über diese Vorgänge wenig bekannt. Ich habe vor einiger Zeit versucht, die „Saitische Formel“ als eine solche Weiheformel für private Tempelstatuen zu erklären, durch 
die der Ka des Besitzers und der jeweilige Gott sich in der Statue verbinden ${ }^{17}$. Eine noch näherliegende Parallele könnte die Weihung einer Situla der Dritten Zwischenzeit sein, die beim Dekadenfest, also im Kult, benutzt wurde. Auf einem solchen Bronzegefäß aus einer Privatsammlung ${ }^{18}$ sind drei Männer vor Amun und anderen thebanischen Göttern dargestellt, die beiden ersten anbetend, der dritte ein Henkelgefäß hochhebend. In der Rede darunter sagt der Stifter, dass er dieses Gefäß für Amun hergestellt habe, um dafür Leben, Heil, Gesundheit und eine schöne Lebenszeit zu erbitten ${ }^{19}$. Vermutlich handelt es sich hier um den Akt der „Weihung“, bei dem der Stifter das Gefäß dem Gott (vermutlich bei einem Fest) präsentierte. Auf einer Situla in einer anderen Privatsammlung ${ }^{20}$ gibt es eine fast identische Darstellung und Inschrift, allerdings ohne den Mann, der die Situla präsentiert. Die Inschrift macht aber auch so klar, was gemeint ist. Eine vergleichbare Szene, hier wieder mit Darstellung des Vorganges, findet sich auf Bronzespiegeln der 25.-26. Dynastie ${ }^{21}$. Die jeweilige Stifterin, eine Gefolgsdame der Mut, die in der Stifungsformel darunter genannt wird, präsentiert den Spiegel der Göttin. Fraglich ist in diesen Fällen ${ }^{22}$ allenfalls, ob der Stifter oder die Stifterin tatsächlich selbst den Gegenstand der Gottheit präsentierte oder ob diese Darstellung nur den ganzen Vorgang in einem Bild zusammenfaßt. In jedem Fall wird man sich bei diesem Schminkgefäß eine ganz ähnliche Weihezeremonie vorzustellen haben. Der Text ist allerdings nicht in der 1. Person gehalten und er wird nicht von dem Stifter gesprochen, sondern von einem anonymen Sprecher. Man darf vielleicht daraus schließen, dass der Stifter zusammen mit einem Priester vor die Göttin trat und der Priester die Zeremonie vollzog und dabei um eine Begünstigung des Stifters bat.

Diese Göttin ist Sachmet, die aber in Kol. 5 als Hathor genannt wird. Beide werden in der 2. Person Singular angesprochen, und es ist klar, dass ein und dieselbe Göttin gemeint ist. Es ist wohlbekannt, dass Sachmet auch als Hathor auftreten kann ${ }^{23}$, beide Göttinnen sind nicht zuletzt als Verkörperung des Sonnenauges verbunden. Es ist

17 SAK 28, 2000, 103-6.

18 S. Schoske; D. Wildung, Gott und Götter im Alten Ägypten, Mainz 1992, 26-7.

19 Jansen-Winkeln, Discussions in Egyptology 32, 1995, 57-62.

20 R.S. Bianchi, Ch. Ziegler, Les Bronzes égyptiens. Fondation Gandur pour l'art, Bern 2014, 146-7 (38); 270 (oben).

21 P. Munro, „Eine Gruppe spätägyptischer Bronzespiegel“, ZÄS 95, 1969, 92-109 (bes. 92-4); Taf. 2-9.

22 Es ist im Übrigen gut möglich, dass auch für das Begräbnis bzw. das Grab bestimmte Figuren vorher im Tempel „geweiht“ wurden, vgl. Jansen-Winkeln, ZÄS 128, 2001, 140-141.

23 Vgl. S.-E. Hoenes, Untersuchungen zu Wesen und Kult der Göttin Sachmet, Bonn 1976, 176-179 immerhin recht wahrscheinlich, dass die an erster Stelle genannte Sachmet die „zuständige“ Ortsgöttin war. Dann wäre die nächstliegende Möglichkeit, dass dieses Gefäß aus Memphis kam.

\section{Der Titel $m r$ mnf3t / mnfyt}

Der Besitzer des Gefäßes war eine Person von hohem Rang, wie der Titel $r p^{c} t h{ }^{3} t j-{ }^{c}$ verrät, aber sein einziger Funktionstitel ist $m r m n f(y) t$.

Das Wörterbuch ${ }^{24}$ definiert $m n f 3 t / m n f y t^{25}$ als „Art Soldaten“, sowohl im allgemeinen Sinne als auch in der engeren Bedeutung von „Fußtruppen“ (im Gegensatz zu Wagenkämpfern). Diese Bestimmung hat viel für sich und man ist ihr im großen und ganzen gefolgt; die häufigste Übersetzung für $m n f 3 t$ ist bis heute „Infanterie“.

Die Bezeichnung und die damit gebildeten Titel sind verschiedentlich untersucht worden, von C. Vandersleyen ${ }^{26}$, A. Schulman ${ }^{27}$ und A. Gnirs ${ }^{28}$, und P.-M. Chevereau hat sie in seine Sammlungen der militärischen Titel aufgenommen und zu klassifizieren versucht ${ }^{29}$.

Mnf3t / mnfyt ist sehr viel seltener als das vielfach gleich oder ähnlich gebrauchte $m \check{s}^{c}$, was natürlich die Bestimmung nicht erleichtert. Zudem wird eine genauere Eingrenzung des Begriffes dadurch erschwert, dass es kaum klare Belege für eine spezifische Verwendung dieser Truppe gibt.

Selbst dass es überhaupt eine (militärische) „Truppe“ ist, wurde von Vandersleyen bestritten. Er hält mnf3t für eine Bezeichnung des Teils der Oberschicht („l'intelligenzia“), aus der die zivile und militärische Füh-

24 Wb II, 80, 1-5.

25 Das Wort wird im Alten und Mittleren Reich durchgehend $m n f 3 t$ geschrieben, im Neuen Reich fast ausnahmslos mnfyt (mnf3t nur in Urk IV, 259,15) und in der Spätzeit meist $m n f(t)$ u. ä.

26 C. Vandersleyen, Les Guerres d’Amosis, MRE I, Brüssel 1971, 176191.

27 A.R. Schulman, Military Rank, Title, and Organization in the Egyptian New Kingdom, MÄS 6, Berlin 1964, 13-14 (§9-12); 40-41 (§93).

28 A.M. Gnirs, Militär und Gesellschaft. Ein Beitrag zur Sozialgeschichte des Neuen Reiches, SAGA 17, Heidelberg 1996, 12-17.

29 P.-M. Chevereau, „Contribution à la prosopographie des cadres militaires de l'Ancien Empire et de la Première Période Intermédiaire“, RdE 38, 1987, 13-47; 40, 1989, 3-36; „Contribution à la prosopographie des cadres militaires du Moyen Empire“, RdE 42, 1991, 43-88; 43, 1992, 11-34; Prosopographie des cadres militaires égyptiens du Nouvel Empire, Antony 1994; Prosopographie des cadres militaires égyptiens de la Basse Epoque, Antony 1985 (263-4); „Addendacorrigenda à la prosopographie des cadres militaires égyptiens de la Basse Epoque“, RdE 41, 1990, 223-230. 
rung rekrutiert wurde ${ }^{30}$. Diese kühne These, mit wenig überzeugenden Argumenten vorgebracht, ist allerdings von kaum jemandem akzeptiert worden. Es ist zu offensichtlich, dass die $m n f 3 t$ Soldaten sind und dass der $m r$ $m n f 3 t$ ein hoher Militärführer ist ${ }^{31}$ : Im Onomastikon des Amenope etwa steht mnfyt zwischen $t z w p d w t$ und $t 3-n t-$ $h t^{3} j^{32}$, und der Titel $z \check{s}$ mnfyt zwischen $m r m \check{s}^{c}$ und jdnw $n$ p3 $m \check{s}^{\mathrm{c3}}$. Teilweise wird mnfyt sogar im bewußten Gegensatz zu nicht-militärischen Gruppen gebraucht ${ }^{34}$, wie schon das Wörterbuch festgestellt hatte ${ }^{35}$. Auch die anderen im Wörterbuch sowie bei Schulman und Gnirs angeführten Beispiele zeigen jenseits jeden Zweifels, dass die mnfyt eine militärische Truppe sind (die aber natürlich auch zu anderen Arbeiten herangezogen werden konnte). Schulman übersetzt $m r$ mnfyt mit „Army officer“ (und $m r$ $m \check{s}^{c}$ mit „Military officer"), Chevereau gibt es durch „chef des troupes “ wieder ( $m r m \check{s}^{c}$ dagegen mit „général“), Gnirs hält beide Titel für Synonyme, und auch in der sonstigen Literatur gibt es kaum Zweifel an der militärischen Natur der mnf3t.

Aus dem Alten und Mittleren Reich kennt man die $m r$ $m n f 3 t$ hauptsächlich als Leiter von Expeditionen ${ }^{36}$. Die wenigen Belege ${ }^{37}$ lassen ansonsten kaum Aussagen zu. Eine Inschrift, die „Annalen“ Amenemhets II. aus Memphis, zeigt immerhin sehr deutlich, dass ein $m r m n f 3 t$ der Oberkommandierende eines Feldzugs (nach Asien) sein konnte ${ }^{38}$.

Im Neuen Reich gibt es eine Reihe von Hinweisen darauf, dass $m r m n f y t$ und $m r m \check{s}^{c}$ nahezu synonym ge-

30 Vgl. Vandersleyen, Les Guerres d'Amosis, 190.

31 Wenn der Titel in einigen Fällen der 18. Dynastie (von denen Vandersleyen ausging) mit dem „Würdenträger“ statt mit dem „Soldaten“ determiniert wird, zeigt das nur, dass der $m r m n f 3 t$ ein hoher Rang war.

32 A.H. Gardiner, Ancient Egyptian Onomastica, London 1947, pl. IX, 15-16 (Nr. 234-237). Dieselbe Abfolge in der Karnak-Inschrift des Merneptah, s. KRI IV, 7,10-11.

33 Gardiner, op. cit., pl.VII,14-VIII,1 (Nr. 87-89).

34 Urk IV, 141,1-2: rhyt ḥr hjj hnw mnfyt ḥr ršrš; KRI II, 320,9: dd.j n.tn rmtw nb srw hrjw t3 mnfyt mj qd.s.

$35 \mathrm{~Wb}$ II, 80, 2.

36 Vgl. Vandersleyen, Les Guerres d'Amosis, 178-9; Chevereau, RdE 38, 1987, 23; Gnirs, Militär und Gesellschaft, 12; D. Stefanović, The Holders of Regular Military Titles in the Period of the Middle Kingdom: Dossiers, London 2006, 207-8. Der Titel eines $m r m \breve{s}^{c}$ ist in den Expeditionsinschriften (auch für den Expeditionsleiter) im übrigen sehr viel häufiger bezeugt, s. E. Eichler, Untersuchungen zum Expeditionswesen des ägyptischen Alten Reiches, GOF IV.26, Wiesbaden 1993, 221-234.

37 Zwei im Alten, sieben im Mittleren Reich, s. Chevereau, RdE 38, 23; 42, 56-7; Stefanović, loc. cit.

38 H. Altenmüller, A. Moussa, SAK 18, 1991, 7; 12; 18 (Kolumnen M 8 ; $16 ; 25)$. braucht werden können; jedenfalls erscheinen sie als Alternative in paarweisen Phrasen ${ }^{39}$. Diese Tatsache wird auch in den einzelnen Studien betont, z. B. von Schul$\operatorname{man}^{40}$ oder Chevereau ${ }^{41}$, und Gnirs überschreibt den entsprechenden Abschnitt in ihrer Arbeit sogar mit „Der Titel jmj-r3 mnfy.t - ein Synonym zum Titel des Heerführers“42. Auch das Wörterbuch versteht ja mnf3t als „allgemein die Soldaten, die Truppen“, also ebenso wie $m \check{s}^{c}$ („Heer, Truppen“) ${ }^{43}$. Im Dekret von Rosette entspricht mnfyt in der „mittelägyptisch“-hieroglyphischen Version einem $p 3 m^{2}{ }^{c}$ in der demotischen ${ }^{44}$.

Andrerseits kann mnfyt auch gebraucht werden, indem es einen von den Wagentruppen unterschiedenen militärischen Verband bezeichnet ${ }^{45}$. Derartige Wortpaare haben dazu geführt, dass mnfyt oft, ja nahezu überwiegend als „Infanterie“ verstanden und übersetzt worden ist ${ }^{46}$. Aber auch $m \check{s}^{c}$ kann ja in dieser Weise neben bzw. im Gegensatz zu $n t$ - $h$ trj verwendet werden ${ }^{47}$, weshalb das

39 Z. B. Urk IV, 911,5-6 und 1459,19: st 3 wrw nw mnfyt ${ }^{\complement} n h w$ nw ms ${ }^{c} r$ pr-c3; 966,5-6: $m h$-jb n nswt $m$ hrp $m \check{s}^{c}$.f snhp qnbt mnfyt; 975,3: [m] $\check{s}^{c} . j \underline{h r-h ̧ 3 t ~ m n f y t} m$ tpj $n$ mšc; 2158,11: hrjw-tp nw mnfyt ${ }^{c} 3$ nb $n$ mšc; KRI II, 91: jty $m k j ~ m \check{s}^{c}$. $f$... sbtj $n$ mnfyt. $f$ u. ä.

40 Military Rank, 13 (§10): „In the Eighteenth Dynasty it [mnfyt] appears always as an alternative or synonym for $m \check{s}^{c}$ in the broad sense of ,the army' including the chariotry“.

41 Cadres militaires égyptiens du Nouvel Empire, 42: „... ce titre qui n'est probablement qu'une simple variante de celui de $m r m \check{s}^{\text {c }}$.

42 Militär und Gesellschaft, 12 (mit „Heerführer“ gibt sie den Titel $m r m \check{s}^{c}$ wieder).

$43 \mathrm{~Wb} \mathrm{II}, 80,1 ; 155$.

44 Urk II, 175. Auch in Edfou I, 165 (dj.jwr f3w. $k m$ jb n m $\check{s}^{c} . k^{c} 3$ hrjt. $k$ hr mnfyt.k) sind beide Alternativen.

45 Im Onomastikon des Amenemope sind $t z w$ pd $w t$, mnfyt und $t 3 \mathrm{nt}$ htrj aufeinander folgende Einträge (Nr. 234-237), s. Gardiner, Ancient Egyptian Onomastica, I, 112-3*; pl.VII,14-VIII,1, auch im Kriegsbericht des Merenptah in Karnak werden beide nebeneinander genannt (Z.30: [mnf]yt nt-ḥtrj $m$ rht ; Z.45: $\underline{\text { tz }}$ pd $t$ t mnfyt $n t$-ḥtrj, s. KRI IV, 5, 13-14; 7,10-11). In der „Heiratsstele“ Ramses II. werden als Truppen Ramses II. zunächst $m \check{s}^{\complement}$ und $n t$-h htrj genannt, dann thrw, mnfyt und $n t$-htrj (KRI II, 250,10-251,3), und im Libyerkrieg Ramses’ III. sind beide kampfbereit (KRI V, 40,9-11) und gehören dann zu den Siegern: mnfyt.f nt-htrj.f hr nhtw (KRI V, 61,2).

46 Vgl. Wb II, 80, 3; Schulman, Military Rank, 13 (§10); 41 (§93); Gardiner, Ancient Egyptian Onomastica, I, 113*; R Hannig, Großes Handwörterbuch Ägyptisch-Deutsch, Mainz 1997, 339; Spalinger, in: J. Moreno Garcia, Ancient Egyptian Administration, Leiden 2013, 422, n.57 und sonst oft.

47 Z. B. Heiratsstele Ramses' II.: jw mšc nt-htrj srw n ḥm.f $m$ šmsw.s jw.w m šbnw hr m $\check{s}^{c} n t$-ḥtrj $n$ Ht3 (KRI II, 250,10-15); Ramses II. in

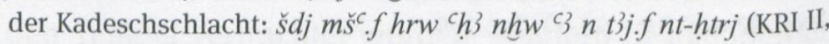
10,1-4);: spdd.n hmm.f p3j.f mšc t3j.f nt-htrj (KRI II, 11,1-4); mšc f nt-

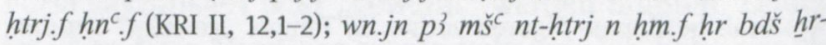

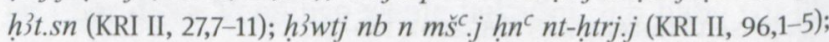
Merneptah im Libyerkrieg: $p 3 m \check{s}^{c} n$ hm.f $h n^{c} n t-h$ trj. $f$ (KRI IV, 6,1-2); Seevölkerkrieg Ramses' III.: $d d-<m d w>$ jn srw smrw hỉwtjw $n$ mšc 
Wörterbuch bei $m \check{s}^{c}$ ebenso die Bedeutung ,das Fussvolk (im Gegs. zu den Wagenkämpfern)“ vermerkt ${ }^{48}$. Die Annahme, „Infanterie“ sei die eigentliche Bedeutung von $m n f y t$, läßt sich von daher kaum rechtfertigen, erst recht nicht in dem Sinne, $m \check{s}^{c}$ sei der Oberbegriff für die bewaffneten Landstreitkräfte und mnfyt die Infanterie im Gegensatz zur Wagentruppe ${ }^{49}$. Chevereau hat außerdem zurecht geltend gemacht, dass es im Alten und Mittleren Reich gar keine Wagentruppe gab, sondern nur „Infanterie“; ein Spezialausdruck dafür wäre daher sinnlos ${ }^{50}$. Im Neuen Reich sei dagegen der Titel $m r$ mnfyt so selten, dass man nicht annehmen könne, es handele sich dabei um den Befehlshaber der Infanterie, die immerhin die Masse des ägyptischen Heeres ausmache ${ }^{51}$.

Die wenigen Belege der 3. Zwischenzeit sind nicht ganz einheitlich. In der 21. Dynastie wird der Hohepriester (und König) Pajnedjem I. in Medinet Habu einmal als $m r$ mnfyt bezeichnet ${ }^{52}$, in derselben Abfolge von Titeln aber sonst als $m r m \check{s}^{c} w r^{53}$, und das ist auch sein üblicher militärischer Titel. In der 22. Dynastie nennt sich ein Militärführer auf derselben Statue einmal $m r$ mnfyt hrrj pdwt $p r-c_{3}$ und einmal $m r m s^{c} h 3 w t j p d w t p r-{ }^{c} 3^{54}$. Der bekannte Armeeschreiber $D d-D h w t j-j w-f-c_{n} h$ A heißt auf einer Stele in Z.1 $m r m \check{s}^{c}(n t) \check{s} m$ ) und in Z.10 derselben Stele $m r$ $m n f y t^{55}$; als $m r m \check{s}^{c}$ ist er noch ein weiteres Mal belegt ${ }^{56}$. In einem Fall sieht es sogar so aus, als sei ein Offizier namens $\underline{D} d-B 3 s t t-j w . f-c_{h} h$ vom $m r m \check{s}^{c}$ zum $m r$ mnfyt befördert worden ${ }^{57}$, aber das dort genannte Amt des $m r$ mnfyt

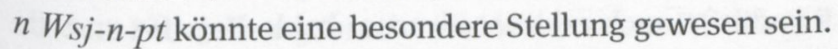

In der 26. Dynastie ist der Titel $m r m n f(y t)$ außer bei Dhwtj-m-3ht noch bei mehr als zehn weiteren Personen belegt $\mathrm{t}^{58}$. Angesichts der Häufigkeit hoher Offiziere und militärischer Titel gerade in dieser Zeit ist das allerdings

nt-ḥtrj ... (KRI V, 27,15); im Papyrus Harris I (75,1) spricht der König zu den srjw ḩ3wtjw nw t3 $m \check{s}^{c} n t-h t_{r j} \check{S} r d n$ pd

$48 \mathrm{~Wb}$ II, 155,4

49 So Schulman, Military Rank, 13 (§10); 41.

$50 \operatorname{RdE} 38,1987,23$.

51 Chevereau, Cadres militaires égyptiens du Nouvel Empire, 42.

52 LD III, 251e; Jansen-Winkeln, Inschriften der Spätzeit, I, 19 $(3.24, \mathrm{c})$.

53 Ibid., 19-20 (3.25)

54 Kairo JE 46600, s. Jansen-Winkeln, Inschriften der Spätzeit, II, 418-20 (45.47).

55 Ibid., 90-1 (17.12); SAK 22, 1995, 180.

56 Kairo CG 42232, j, s. Jansen-Winkeln, Inschriften der Spätzeit II, 207.

57 Ibid., 421 (45.50, Rückseite, Z.2-3); vgl. Jansen-Winkeln, Ägyptische Biographien der 22. und 23. Dynastie, ÄUAT 8, 1985, 304 (mit Anm.3).

58 Pressl, Beamte und Soldaten, 87. nicht besonders viel ${ }^{59}$. Pressl übersetzt mit „Vorsteher der Infanterie "60 und hält die "Gruppe der mnf.yt bezogen auf die der $m \check{s}^{c}$ “ für einen ,weniger bedeutenden militärischen Verband“61. Das ergibt sich aber eigentlich nur aus ihrer vorgefaßten Meinung, ein $m r m \check{s}^{c}$ müsse „der Oberkommandierende aller ... Truppenteile“62 sein. Tatsächlich gibt es nicht nur einen ${ }^{63}$, sondern mehrere Fälle, wo derselbe Offizier $m r m \check{s}^{c}$ und $m r m n f y t$ ist ${ }^{64}$. Die Personen mit dem Titel $m r$ mnfyt sind jedenfalls sehr hohe Funktionäre ${ }^{65}$, und einige davon gehören zu den bekanntesten dieser Zeit, z. B. Jch-msjw (Amasis) und P3-dj-Zm3-t3wj (Potasimto), die beiden Befehlshaber des Nubienfeldzugs Psametiks II., oder $N s-H r$ und $W 3 h-j b-R^{c}$. Es ist allerdings auffallend, dass diejenigen darunter, die mehrere Denkmäler hinterlassen haben, diesen Titel nicht durchgehend tragen $^{66}$. Es wäre daher denkbar, dass sich dieser Titel nicht auf eine ständige Funktion bezieht, sondern auf eine besondere Gelegenheit, einen Auftrag.

Man kann also soweit nur feststellen, dass mnfyt wie auch $m \check{s}^{c}$ sowohl „Armee, Soldaten“ (schlechthin) bezeichnen können als auch (ab dem Neuen Reich) im engeren Sinne die „Fußtruppen, Infanterie“ im Gegensatz zur Wagentruppe ${ }^{67}$. Beide Bezeichnungen werden in den gleichen Kontexten verwendet und ihre Bedeutung muß demnach nahezu gleich oder jedenfalls sehr ähnlich sein.

59 Der Titel $m r m \check{s}^{c}$ ist etwa viermal so häufig.

60 Beamte und Soldaten, 86.

61 Ibid., 90.

62 Ibid., 86.

63 Ibid., 87.

64 Auf seiner Statue Kairo CG 48637 (JE 36949) ist $\underline{D} d-P t h$-jw. $f$ - $n h$ sowohl $m r m \check{s}^{c}$ (Sockel) als auch $m r m n f y t$ (Vorderseite, Z.5; Rückseite), s. De Meulenaere, BIFAO 63, 1965, 20; 22; 23; W3 $h-j b-R^{c}$ ist auf BM $111 m r m \check{s}^{c} n b$ nw tp rsj mhtj (Jansen-Winkeln, Inschriften der Spätzeit, IV, 490 [57.188]) und auf Kairo JE 34043 mr mnfyt (ibid., 491 [57.190]); Psmtk-nb-phtj nennt auf seinem Sarkophag Kairo TN 21/11/14/6 mehrfach $m r m \check{s}^{c}$ und einmal $m r$ mnf3t (ibid., 911-14 [60.291]); ${ }^{C} n h-H r$ hat den Titel $m r m n f y t$ auf seiner Serapeumstele Kairo JE 43204 (ibid., 922 [60.306]) und auf dem Statuensockel London UC 14629 (ibid., 1143 [60.714]), aber auf letzterem ist er auch ḥrj $n m \check{s}^{c}$.

65 Praktisch alle von ihnen tragen den Rangtitel $r p^{c} t h 3 t j-{ }^{c}$.

66 Der bekannte Grenzoffizier $W 3 h-j b-R^{c}$ führt ihn nur auf seiner Statue Kairo JE 34043 (Jansen-Winkeln, Inschriften der Spätzeit, IV, 57.191), auf weiteren 15 Objekten von ihm dagegen nicht, der Offizier $\mathrm{Ns}$ - $\mathrm{Hr}$ wird nur auf zwei von sieben Denkmälern so bezeichnet (Pressl, Beamte und Soldaten, 224-6).

67 Es ist gerade bei militärischen Bezeichungen nicht selten, dass sie in einem engeren und weiteren Sinn verwendet werden können, auch das moderne „Armee“ z. B. ist mehrdeutig, denn es kann die Gesamtheit der Streitkäfte bezeichnen ebenso wie einen bestimmten Großverband („3. Armee“). Auch Bezeichnungen für Waffen können öfter eine engere und eine weitere Bedeutung haben (im Deutschen z. B. „Kanone“ oder „Geschütz“). 
Dennoch sollte zumindest ursprünglich ein Unterschied vorhanden gewesen sein, und selbst Ramses II. führt einmal in einer langen Aufzählung hoher Funktionäre $m r m \check{s}^{c}$ und $m r$ mnfyt nebeneinander auf ${ }^{68}$, sie sind offenbar auch in der 19. Dynastie noch nicht wirklich identisch $^{69}$. Da der Unterschied über die Kontexte nicht zu ermitteln ist, könnte vielleicht die Etymologie einen Hinweis geben.

Tatsächlich gibt es schon seit langem einen Versuch, die Bedeutung von mnfit/mnfyt mithilfe der Etymologie genauer zu bestimmen: Gardiner hatte in seinem Kommentar zum Onomastikon des Amenope vorgeschlagen, das Wort als $m$-Bildung zu $n f$,wrongdoing“ zu erklären und war dadurch zu der Übersetzung ,aggressors, attackers “ gekommen $^{70}$. Faukner ist dem gefolgt, zieht aber die etwas professioneller klingende Übersetzung „shocktroops“ („Sturmtruppen“ o. ä.) vor ${ }^{71}$. Das ist allerdings kaum überzeugend, weder die wörtliche Bedeutung als „Übeltäter“ noch die übertragene, denn nirgends deutet sich eine derart spezifische Funktion der mnfyt-Truppen an. In nahezu allen Kontexten handelt es sich eher um eine Sammelbezeichnung für Soldaten insgesamt bzw. (allgemein) Fußtruppen im Gegensatz zu den Wagenkämpfern.

Andrerseits ist Gardiners Idee, dass hier eine $m$-Bildung vorliegt, sicher sehr naheliegend ${ }^{72}$, zumal, wenn man von der offenbar älteren Form $m n f 3 t$ ausgeht, denn 4-radikalige nichtreduplizierte Wurzeln sind selten. Nominalbildungen mit $m$-Präfix kommen im Ägyptischen (wie im Semitischen) als nomina loci, nomina instrumenti oder Partizipien vor ${ }^{73}$. Für $m n f 3 t$ käme wohl nur eine Partizipialbildung in Frage ${ }^{74}$.

Geht man von der älteren Schreibung mnf3t aus, müßte das Ableitungsverb bzw. die zugrundeliegende Wurzel $n f 3$ sein. Ein Verb $n f 3$, ausatmen, schnauben“ (etc.) ist in

68 KRI II, 608,10; ausführlich zitiert bei Gnirs, Militär und Gesellschaft, 15-16.

69 Selbst wenn Gnirs damit recht haben sollte, dass dieses Nebeneinander nicht den „aktuellen Stand“ der Verwaltung widerspiegelt, sondern „sich auf eine bereits überholte, aber offensichtlich immer noch als ideal angesehene Amtsverteilung früherer Epochen“ bezieht, müßte dann wenigstens in diesen "früheren Epochen“ $m r$ $m n f 3 t$ von $m r m s^{c}$ unterschieden gewesen sein

70 Ancient Egyptian Onomastica, I, 113*.

71 JEA 39, 1953, 38.

72 In H. Grapows Untersuchung Über die Wortbildungen mit einem Präfix $m$ - im Ägyptischen, APAW 1914, 1-33, wird $m n f 3 t$ nicht erwähnt, aber das ist nicht verwunderlich, da weder Bedeutung noch Ableitungsverb deutlich sind.

73 Vgl. E. Edel, Altägyptische Grammatik, Rom 1955, §253-256.

74 Eine Gruppe Soldaten als „Instrument“ zu betrachten, wäre sicher eine zu abstrakte oder moderne Anschauung. den Sargtexten belegt ${ }^{75}$ und sollte eine (lokale, dialektale?) Variante des viel häufigeren $n f j$,ausatmen, hauchen, blasen ${ }^{\text {"76 }}$ sein. Ein Wechsel von Verben III. inf. mit dreiradikaligen Verben auf -3 ist auch sonst bezeugt ${ }^{77}$. Von $n f j$ sind recht häufige Nomina wie $n f(w)$ „Atem, Wind“ und $n f w(j)$ „Schiffer“ abgeleitet. Man könnte daher erwägen, ob mnfit/mnfyt nicht eine (passive) Partizipialbildung zu $n f 3 / n f j$ „blasen“ sein könnte: „Geblasene“ im Sinne von „Vom Wind Beförderte“78. Dann ergäbe sich als eigentliche Bedeutung von mnf3t „zu Schiff beförderte (Soldatentruppe)“79.

Die beiden elementaren Arten der Fortbewegung im Alten Ägypten, jedenfalls vor der Einführung des Wagens, waren das Gehen bzw. Laufen und die Fahrt auf dem Wasser $^{80}$. Das Wort $m \check{s}^{\complement}$ „Armee, Truppe, Soldaten“ hängt natürlich mit $m s ̌ c j$ „gehen, marschieren“ zusammen. Da dieses Verb erst recht spät belegt ist, könnte man es für denominal halten ${ }^{81}$, aber J. Zeidler hat vor einiger Zeit dargelegt, dass tatsächlich eher das Nomen „Armee“ vom Verb „marschieren“ abgeleitet ist ${ }^{82}$. Also wäre die $m \check{s}^{\mathrm{C}}$-Truppe als „Marschierer“ dem Wortsinne nach die eigentliche „Infanterie“. Wenn die oben vorgeschlagene Ableitung richtig ist, waren die $m n f 3 t$ dann die zu Schiff transportierten Soldaten. Natürlich mußten beide „zu Fuß“ kämpfen und sicher hatten auch die mit Schiffen oder Booten angelandeten Soldaten noch zu „marschieren“. Der Unterschied betrifft also nur den Transport vor dem Kampf und erfordert keinen Unterschied in Ausbildung oder Bewaffnung. Das dürfte auch erklären, warum beide Begriffe und die entsprechenden Titel wie Synonyme verwendet werden können. Das sehr viel häufigere $m \check{s}^{c}$ dürfte von Anfang an die normale, übliche Bezeichnung für Soldaten gewesen sein.

Die Notwendigkeit, überhaupt eine zweite Bezeichnung einzuführen, könnte sich ergeben haben, wenn ein Teil der Truppen auf dem Landweg vorrückte und der

$75 \mathrm{~Wb}$ II, 252, 3; R. van der Molen, A Hieroglyphic Dictionary of Egyptian Coffin Texts, PÄ 15, Leiden 2000, 222; Osing, MDAIK 43, 1987, $209(n f 3-h r)$.

76 Wb II, 250-1; J. Osing, Die Nominalbildung des Ägyptischen, Mainz 1976, 82; 544-5 (394).

77 U.a. $h w 3$ „werfen“ und $h w j$ „schlagen, werfen“; $h b 3$ und $h b j$ „schmälern“, $j \underline{t} 3$ ubd $j t j$,wegnehmen“, $n g$ 3 und $n g j$ „,brechen“ u. a.m. 78 Das wäre sicher nicht merkwürdiger als die Bezeichnung des Schiffers oder Matrosen als „Zum Wind Gehöriger“, s. Osing, Nominalbildung, 317 (Nisbe von $n f w$,Wind“).

79 Bei der späteren Form mnfyt wird man wohl an die Endung der Kollektiva gedacht haben.

$\mathbf{8 0}$ Reiten auf Eseln ist kaum belegt und spielte jedenfalls für das Militär keine Rolle.

81 So z. B. Goedicke, SAK 25, 1998, 108.

82 WdO 29, 1998, 25-26. 
andere per Schiff transportiert wurde, wie das in der Inschrift des $W n j$ bezeugt ist ${ }^{83}$, aber sicher auch sonst öfter vorgekommen sein wird. Da in Ägypten vermutlich alle Truppen soweit wie möglich per Schiff transportiert wurden, könnte diese Bezeichnung dann bald nur zu einer (selteneren) Alternative für $m \check{s}^{c}$ geworden sein ${ }^{84}$.

Die Frage ist allerdings, ob es wirklich nur noch eine Art Variante war oder ob (und wie lange) auch die eigentliche Bedeutung bei der Wahl dieser Bezeichnung eine Rolle spielte. In den meisten Fällen dürfte das kaum zu beantworten sein, da der Nil, die Nilarme oder das Meer bei militärischen Operationen in oder außerhalb Ägyptens fast immer (auch) als Transportweg in Frage kommen. Zumindest bei dem Feldzug nach Asien, der in den Annalen Amenemhets II. verzeichnet ist, könnte $m n f 3 t$ noch seine eigentliche Bedeutung haben, denn offenbar wurden die Truppen per Schiff dorthin transportiert ${ }^{85}$. Einen Hinweis darauf, dass mnfyt im engeren Sinne die zu Schiff transportierten Soldaten sind, ist vielleicht auch darin zu sehen, dass sie (relativ) häufig bei Expeditionen und Bauaktivitäten zum Ensatz kamen. Und das ist auch noch im Neuen Reich so: Als Ramses II. zu Beginn seiner Regierung Bauarbeiten in Abydos plant, läßt er neben dem Hofstaat die Generäle der mnfyt-Truppen, die Baumeister und die Archivare zusammenrufen ${ }^{86}$. Die letzteren beiden Gruppen werden für Planung und Bau zuständig sein, die Generäle vermutlich für den Transport des Materials, das zu Schiff herangeschafft und dann von den Soldaten $\mathrm{zu}$ den Baustellen gebracht werden konnte. Für die Bewachung der Baustelle hätte man sicher keine größeren Truppenteile und mehrere Generäle benötigt. Im Papyrus Anastasi I (13.5-7) ist von einem Befehlsschreiber (zš sḥn) der mnfyt-Truppen die Rede, der den Auftrag erhält, einen See zu graben und die Rationen der Truppen zu errechnen $^{87}$. Die besondere Beziehung der mnfyt-Soldaten und

\section{Urk I, 104-5.}

84 Falls die vorgeschlagene Ableitung richtig ist, wäre es an sich naheliegend, $m n f 3 t$ durch „Marineinfanterie“ wiederzugeben. Aber solch eine moderne Bezeichnung könnte leicht zu falschen Vorstellungen führen. Ägyptische Soldaten, die zu Schiff (auf dem Nil oder in Küstennähe auf dem Mittelmeer) transportiert wurden, waren kaum eine besonders ausgebildete Truppe und auch nicht speziell für Einsätze in „Übersee“ gedacht.

85 Das entscheidende Wort $m 3^{c}$,aussenden“ in M.8 ist mit dem Schiff determiniert. Vermutlich waren auch die beiden auf dem Feldzug angegriffenen Orte nur auf dem Seeweg erreichbar, s. jetzt H. Altenmüller, Zwei Annalenfragmente aus dem frühen Mittleren Reich, BSAK 16, 2015, 306.

86 „Inscription dédicatoire“, Z.38, s. KRI II, 326,6-7.

87 H.-W. Fischer-Elfert, Die satirische Streitschrift des Papyrus Anastasi I, ÄgAb 44, Wiesbaden 1986, 119-20; Textzusammenstellung, ${ }^{2} 1992,106-107$. ihrer Befehlshaber zu Bautätigkeiten würde sich leicht erklären, wenn es sich um eine Truppe handelte, die für den Transport zu Schiff bestimmt war. Bei den militärischen Titulaturen in der 26. Dynastie ist es zumindest auffällig, dass gerade die Generäle sich auch als $m r$ mnfyt bezeichnen, die unter Psametik II. den Nubienfeldzug befehligten ${ }^{88}$, bei dem die Truppen wohl in der Hauptsache zu Schiff transportiert wurden ${ }^{89}$.

Über Person und Aktivitäten des Generals $\underline{D} h w t j-m$ $3 h t$, der das hier besprochene Gefäß gestiftet hat, ist allerdings weiter nichts bekannt, und so wissen wir auch nicht, ob sein Titel $m r$ mnfyt hier nur eine Art Synonym zum üblicheren $m r m \check{s}^{c}$ war oder ob er tatsächlich eine zu Schiff transportierte Truppe befehligt hat.

Acknowlwdgement: Ich danke Rachel Barclay vom Oriental Museum in Durham sehr herzlich für Fotos und andere Informationen über das Gefäß und seine Inschrift.

\section{Bibliographie}

Ahrens, A., 2006, „A Journey’s End: Two Egyptian Stone Vessels with Hieroglyphic Inscriptions from the Royal Tomb of Tell Mišrife/ Qaţna“, Ä\&L 16, 15-36.

Altenmüller, H.; Moussa, A., 1991, „Die Inschrift Amenemhets II. aus dem Ptah-Tempel von Memphis. Ein Vorbericht“, SAK 18, 1-48.

Altenmüller, H., 2015, Zwei Annalenfragmente aus dem frühen Mittleren Reich, BSAK 16.

Aston, B., 1994, Ancient Egyptian Stone Vessels. Materials and Forms, SAGA 5, Heidelberg.

Becker, M., 2012, Identität und Krise, BSAK 13, Hamburg.

Bianchi, R.S., Ziegler, C., 2014, Les Bronzes égyptiens. Fondation Gandur pour l'art, Bern.

Birch,S., 1880, Catalogue of the Collection of Egyptian Antiquities at Alnwick Castle, London.

Bongrani Fanfoni, L., 1987, „Un nuovo documento di Scepenupet la e Amenardis la“, OrAnt 26, 65-71.

Careddu, G., 1985, Museo Barracco di scultura antica. La collezione egizia, Rom.

Chevereau, P.M. 1985, Prosopographie des cadres militaires égyptiens de la Basse Époque, Antony.

Chevereau, P.M. 1987, „Contribution à la prosopographie des cadres militaires de l'Ancien Empire et de la Première Période Intermédiaire“, RdE 38, 13-47.

88 Der General Jch-msjw auf der Statue Kairo CG 895 + New York MMA 66.99.68, s. Jansen-Winkeln, Inschriften der Spätzeit, IV, 395 (56.123), der General P3-dj-Zm3-t3wj auf dem Gefäß Kairo CG 18736, ibid., 847 (60.199).

89 Sie sind nach Süden vorgestoßen „soweit der Fluß es zuließ“, s. P. Haider, „Epigraphische Quellen zur Integration von Griechen in die ägyptische Gesellschaft der Saitenzeit“, in: U. Höckmann / D. Kreikenbom (Hgg.), Naukratis. Die Beziehungen zu Ostgriechenland, Ägypten und Zypern in archaischer Zeit, Paderborn 2001, 204; 212. 
Chevereau, P.M. 1990, „Addenda-corrigenda à la prosopographie des cadres militaires égyptiens de la Basse Epoque“, RdE 41, 223-230.

Chevereau, P.M. 1991, „Contribution à la prosopographie des cadres militaires du Moyen Empire“, RdE 42, 43-88.

Chevereau, P.M., 1994, Prosopographie des cadres militaires égyptiens du Nouvel Empire, Antony.

De Meulenaere, 1965, „La statue du général Djed-ptah-iouf-ankh, (Caire JE 36949) “, BIFAO 63, 19-32.

Edel, E., 1955, Altägyptische Grammatik, Rom.

Eichler, E., 1993, Untersuchungen zum Expeditionswesen des ägyptischen Alten Reiches, GOF IV.26, Wiesbaden.

el-Khouli, A. 1978, Egyptian Stone Vessels, predynastic period to dynasty III, Mainz.

Fischer-Elfert, H.-W, 1986, Die satirische Streitschrift des Papyrus Anastasi I, ÄgAb 44, Wiesbaden.

Gardiner, A.H., 1947, Ancient Egyptian Onomastica, London 1947.

Gnirs, A.M., 1996, Militär und Gesellschaft. Ein Beitrag zur Sozialgeschichte des Neuen Reiches, SAGA 17, Heidelberg.

Goedicke, H. 1998, „Dienstränge im Alten Reich?“, SAK 25, 101-111.

Grapow H. 1914, Über die Wortbildungen mit einem Präfix m- im Ägyptischen, APAW.

Günther, P.; Wellauer, R. 1988, Ägyptische Steingefäße der Sammlung Rudolph Schmidt Solothurn, Zürich.

Haider, P., 2001, „Epigraphische Quellen zur Integration von Griechen in die ägyptische Gesellschaft der Saitenzeit“, in: U. Höckmann, D. Kreikenbom (Hgg.), Naukratis. Die Beziehungen zu Ostgriechenland, Ägypten und Zypern in archaischer Zeit, Paderborn, 197-215.

Hannig, R. 1997, Großes Handwörterbuch Ägyptisch-Deutsch, Mainz.

Hodjash, S. 2005, Ancient Egyptian Vessels in the State Pushkin Museum of Fine Art, Moscow, Baltimore.

Hoenes, S.E., 1976, Untersuchungen zu Wesen und Kult der Göttin Sachmet, Bonn.

Jansen-Winkeln, K. 1985, Ägyptische Biographien der 22. und 23. Dynastie, ÄUAT 8, Wiesbaden.

Jansen-Winkeln, K., 1995, „Bezeichnung und Funktion einer Situla“, Discussions in Egyptology 32, 1995, 57-62.

Jansen-Winkeln, K. 1995, „Neue biographische Texte der 22./23. Dynastie“, SAK 22, 1995, 169-194.

Jansen-Winkeln, K. 2001, „Eine Familie im Totenkult“, ZÄS 128 , 133-141.

Jansen-Winkeln, K., 2007, Inschriften der Spätzeit Teil II: Die 22.-24. Dynastie, Harrassowitz, Wiesbaden.

Jansen-Winkeln, K. 2007, Inschriften der Spätzeit Teil I: Die 21. Dynastie,

Jansen-Winkeln, K., 2014, Inschriften der Spätzeit. Teil IV: Die 26. Dynastie, Wiesbaden.
Kayser, H. 1969, Ägyptisches Kunsthandwerk, Braunschweig. Kitchen, K. 1969-1990, Ramesside Inschription, Oxford.

Klemm, R. und D. 1981, Die Steine der Pharaonen, München.

Lacovara, P. 1997, „Rezension zu Lilyquist, Egyptian Stone Vessels: Khian through Thutmosis IV“, AJA 101, 780-782.

Lauer, J.-P. 1934, „Fouilles du Service des Antiquites a Saqqarah

(Secteur Nord) (Novembre 1933-Mai 1934) “, ASAE 34, 65-67.

Lauer, J.-P. 1939, La pyramide à degrés III, Kairo.

Lauer, J.-P, 1959, La pyramide à degrés IV, Kairo.

Lauer, J.-P., 1965, La pyramide à degrés V, Kairo.

Lauer,J.-P. 1962, Les pyramides à degrés, BdE 39, Kairo, 91-98.

Lilyquist, C. 1995, Egyptian Stone Vessels. Khian through Thutmosis IV, New York.

Meffre, R. 2015, D’Hérakléopolis à Hermopolis, Paris.

Munro, P. 1969, „Eine Gruppe spätägyptischer Bronzespiegel“, ZÄS 95, 92-109.

Osing, J. 1976, Die Nominalbildung des Ägyptischen, Mainz 1976.

Osing,J. 1987, “Sprüche gegen die $j b h 3 t j$-Schlange, MDAIK 43, 205-210.

Pommerening, T. 2005, Die altägyptischen Hohlmaße, Beihefte SAK 10 , Hamburg.

Posener, G. 1936, La première domination perse en Egypte, BdE 11, Kairo 1936.

Pressl, D. 1998, Beamte und Soldaten. Die Verwaltung in der 26. Dynastie in Ägypten (664-525 v. Chr.), Frankfurt a. M.

Schoske, S., Wildung, D. 1992, Gott und Götter im Alten Ägypten, Mainz.

Schulman, A.R. 1964, Military Rank, Title, and Organization in the Egyptian New Kingdom, MÄS 6, Berlin.

Shaw, I. 2010, „Inscribed Stone Vessels as Symbols of the EgyptoAchaemenid Economic Encounter“, in: A. Hudecz, M. Petrik (edd.), Commerce and Economy in Ancient Egypt, Oxford, 113-117.

Sist, L. 1996, Museo Barracco. Arte egizia, Rom.

Spalinger, A. 2013, "The Organisation of the Pharaonic army (Old to New Kingdom)", in: J. Moreno Garcia, Ancient Egyptian Administration, Leiden, 393-478.

Stefanović, D. 2006, The Holders of Regular Military Titles in the Period of the Middle Kingdom: Dossiers, London.

van der Molen, R. 2000, A Hieroglyphic Dictionary of Egyptian Coffin Texts, PÄ 15, Leiden.

Vandersleyen, C. 1971, Les Guerres d'Amosis, MRE I, Brüssel.

Vandier d'Abbadie, J. 1972, Catalogue des objets de toilette égyptiens, Paris.

Vlčková, P. 2006, Stone Vessels from the Mortuary Complex of Raneferef at Abusir (Abusir XV), Prag 2006.

von Bissing, F.W. 1907, Steingefäße, CG, Leipzig.

Zeidler, J. 1998, Beiträge zur Nominalbildung des Ägyptischen, WdO $29,21-32$. 


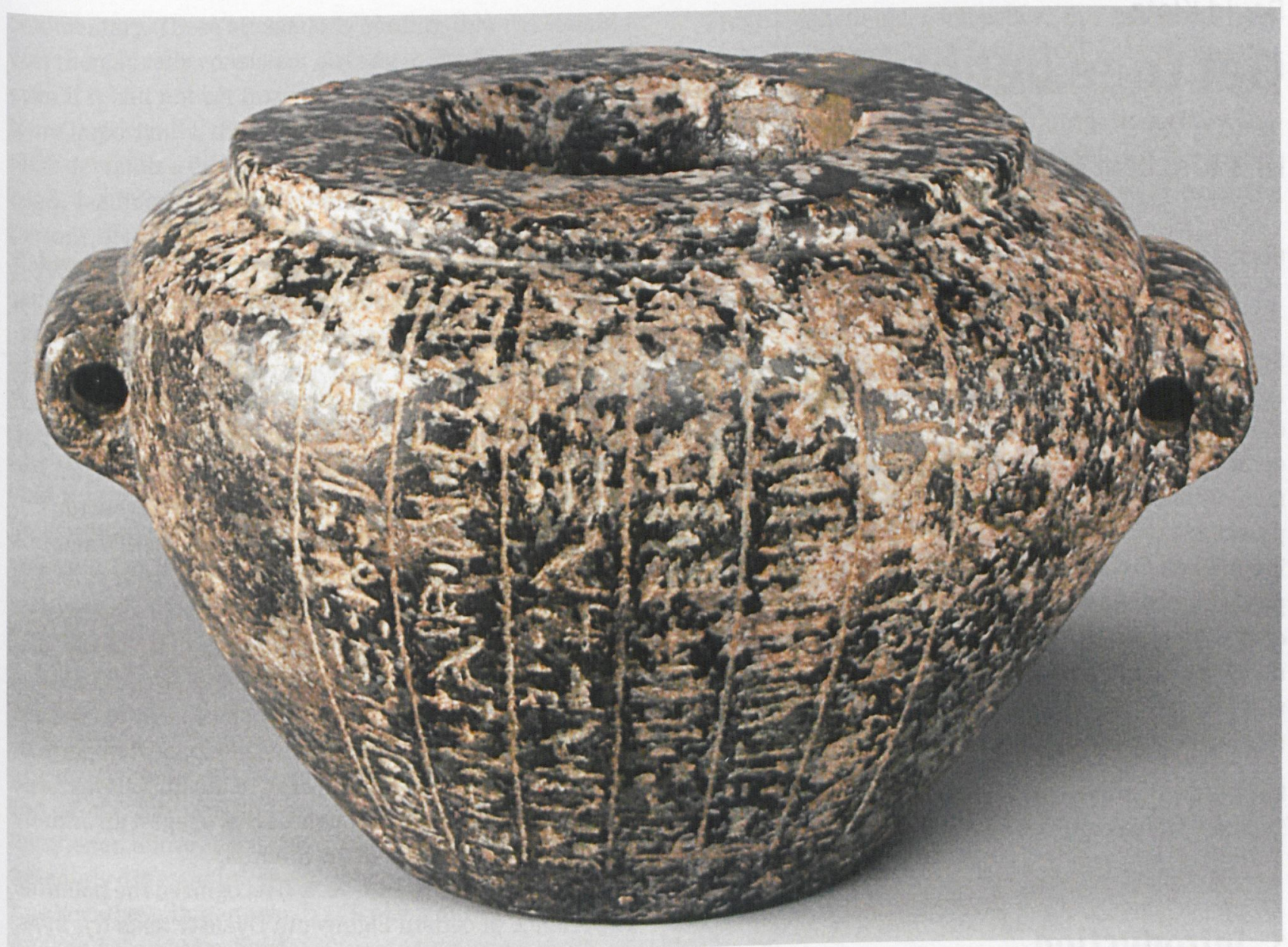

Abb. 2: Schminkgefäß Durham EG3995 mit Inschrift.
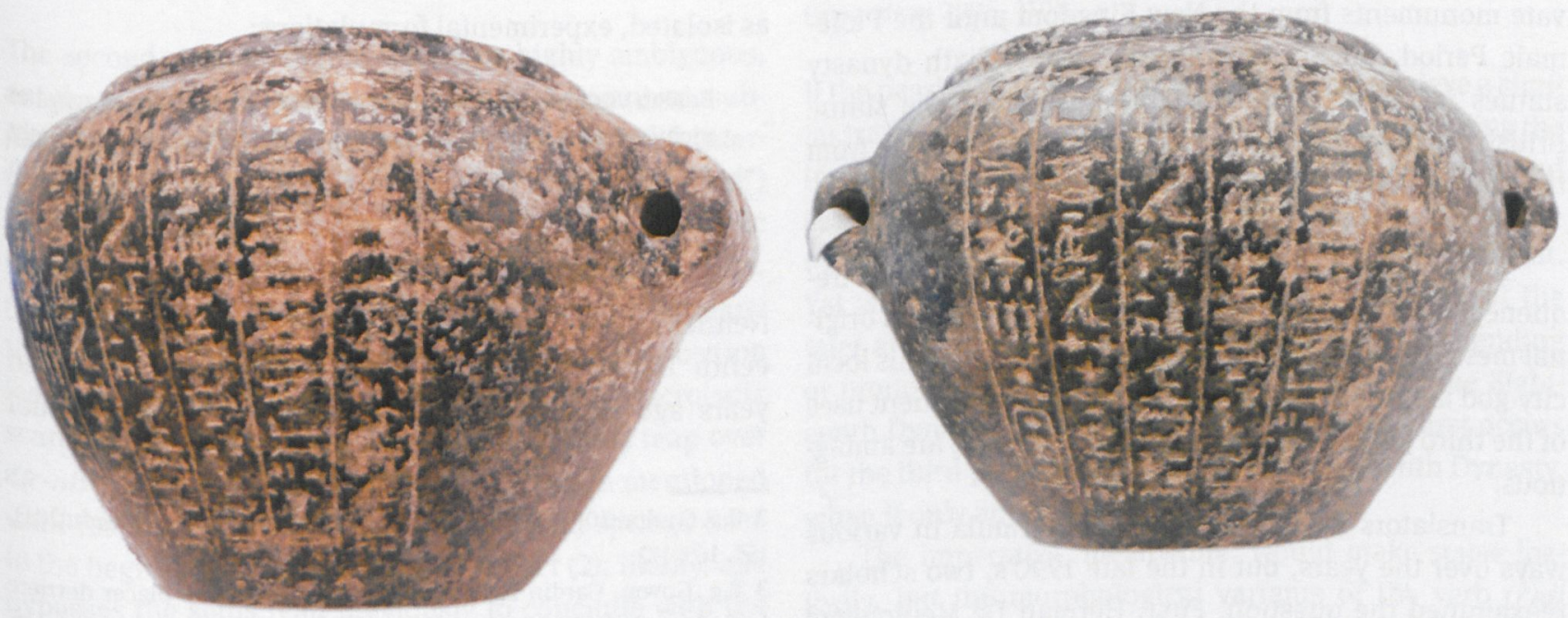

Abb. 3: Detail: Inschrift. 